\title{
Time course of response to oral and inhaled corticosteroids in non-asthmatic chronic airflow obstruction
}

\author{
D C Weir, A S Robertson, R I Gove, P Sherwood Burge
}

\begin{abstract}
One hundred and twenty one patients considered on clinical grounds to have non-asthmatic chronic airflow obstruction completed a double blind, crossover trial comparing oral prednisolone $40 \mathrm{mg}$ per day with inhaled beclomethasone dipropionate $500 \mu \mathrm{g}$ thrice daily, each given for 14 days, with a 14 day washout period between treatments. The time course of response was analysed for the $\mathbf{5 7}$ occasions where there was a significant increase in mean daily peak expiratory flow (PEF) over the treatment period. Mean daily PEF was still rising at day 14 on 12 occasions. After withdrawal of treatment mean daily PEF remained above pretreatment levels for more than two weeks in half the responses analysed. The peak response occurred earlier with inhaled beclomethasone (median 9.5 (range 3-14) days) than with oral prednisolone (median 12 (range 1-14) days), though both treatments produced a response that was sustained for a similar period. The results suggest that a trial of treatment with corticosteroids in this group of patients should last more than 14 days, and that in a study with a crossover design the washout period should be longer than two weeks.
\end{abstract}

It is common practice to give patients with non-asthmatic chronic airflow obstruction a trial of corticosteroids to identify patients in whom clinical improvement occurs. ${ }^{12}$ In such trials the drug is usually given for two to four weeks, but the rationale for this duration of treatment is not clear. ${ }^{3}$ In a group of predominantly asthmatic patients Webb et al showed that those responding to prednisolone did so by the eleventh day of treatment. ${ }^{4}$ No comparable work has been carried out in patients with non-asthmatic airflow obstruction.

In a randomised, double blind crossover trial comparing placebo, inhaled beclomethasone, and oral prednisolone we showed that a response to placebo treatment was more likely to occur when this was preceded by an active treatment period, despite a two week washout period between treatments. ${ }^{5}$ This suggests a carry over effect of active treatment of at least three to four weeks as response to steroid treatment was not measured until four weeks after the previous treatment had been stopped.
We have analysed the time course of the response to oral and inhaled corticosteroids by assessing the time taken to reach a plateau in PEF with treatment in our crossover study ${ }^{5}$ and the time taken for PEF to return to pretreatment levels after cessation of treatment.

\section{Methods}

SUBJECTS

Outpatients with chronic airflow obstruction of at least five years' duration were recruited to the trial. Patients were excluded if they had a clinical diagnosis of asthma. Details are given in the previous article. No patient had received oral or inhaled corticosteroids in the preceding six months. All patients gave informed consent.

\section{DESIGN}

The trial was a randomised, double blind, double dummy crossover comparison of inhaled beclomethasone dipropionate $500 \mu \mathrm{g}$ thrice daily, oral prednisolone $40 \mathrm{mg} /$ day, and placebo. Each treatment phase lasted two weeks and was followed by a two week washout period before the next treatment was given. The first treatment period was preceded by a four week baseline period. Patients attended the laboratory on days 1 and 14 of the baseline period, and on the last day of each treatment period. Fourteen further patients who completed the same protocol without the placebo period are included in this analysis, as response to treatment has been defined without reference to the placebo period in view of the treatment order effect noted in the trial. ${ }^{5}$

\section{MEASUREMENTS}

$\mathrm{FEV}_{1}$ and FVC were measured on two occasions during the baseline phase before any trial treatment was given, and on the final day of each treatment phase, all visits being at the same time of day. Patients were requested to refrain from inhaled bronchodilators for six hours before measurements. The mean of three technically satisfactory blows within $10 \%$ or $100 \mathrm{ml}$ (whichever was the smaller) on a dry wedge (Vitalograph) spirometer was used for analysis. Baseline $\mathrm{FEV}_{1}$ and FVC were taken as the highest mean measurements recorded during the baseline period.

After the second baseline visit patients were asked to measure peak expiratory flow (PEF), using a mini Wright's peak flow meter, four hourly during waking hours at home. They were instructed to record the best of at least three attempts with the highest two readings 
Figure 1 Mean daily $P E F$ in subject 1 , showing a good response to oral prednisolone reaching a peak on the 10th day of treatment (day 17). After the end of treatment the readings show a gradual fall through the following placebo phase, returning to pretreatment levels on the 28th day after treatment was stopped (day 49).

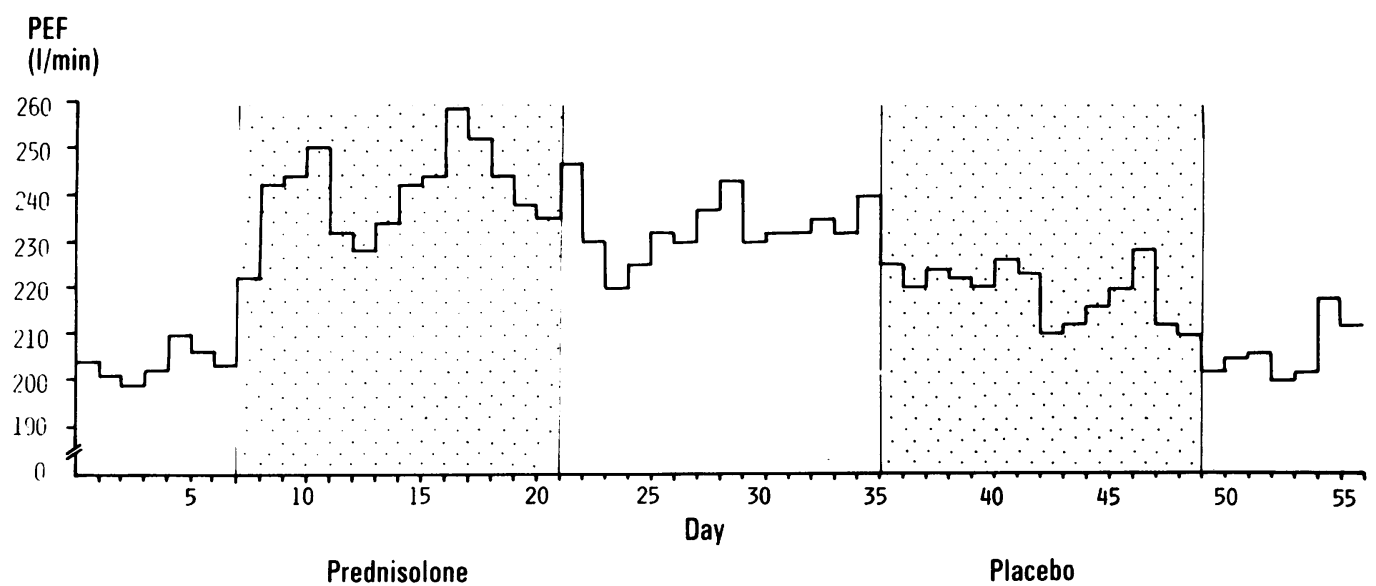

within $20 \mathrm{l} / \mathrm{min}$. Baseline values were obtained during the two weeks before the first treatment phase, and the mean PEF was calculated over the final seven days of this period. Patients were asked to maintain a constant interval between their regular bronchodilator medication and the PEF measurements.

\section{ANALYSIS}

Definition of response to treatment

A response to treatment was assessed by comparison with initial baseline measurements, because a treatment order effect was seen. A full response was defined as an improvement in absolute values of $\mathrm{FEV}_{1}$ or FVC of at least $20 \%$ measured on the last treatment day, or the same improvement in mean PEF over the final seven days of the treatment period, over the values obtained during the baseline period (see preceding article). A partial response was defined as improvement of at least $15 \%$ in one of these measurements or of at least $10 \%$ in any two measurements.

\section{Analysis of peak flow records}

Selection of PEF records For all treatment periods producing a full or partial response (as defined) we analysed PEF records further. Mean PEF on the seven days immediately before the treatment period in which a response was seen (pretreatment period) was compared with mean PEF on the final seven days of treatment by an unpaired Student's $t$ test. Any response showing a significant improvement in PEF ( $5 \%$ level of significance) with treatment by this means was analysed further. This allowed us to analyse records of PEF where response occurred only in $\mathrm{FEV}_{1}$ or FVC but which showed statistically detectable increases in PEF with treatment.

Graphical analysis of selected $P E F$ records In the records showing a significant improvement in PEF mean daily PEF was plotted graphically (fig 1). Two observers assessed independently, using predetermined criteria, $(a)$ the time taken to reach the peak treatment response and $(b)$ the time taken for readings to return to the pretreatment level.

Time to peak response was defined as the day on which the highest daily mean PEF was reached. The data on time to peak effect are censored, as the true value in the 12 responses showing peak daily mean PEF on the final treatment day is unknown. To compare the time to peak effect for the two drugs we have used a ranking test using all the data; excluding these 12 responses gives the same results.

For duration of treatment effect we defined the time taken to reach the pretreatment level as the first day on which mean daily PEF fell below the upper $90 \%$ confidence limit for the mean PEF during the seven pretreatment days, provided that such a fall was sustained for two or more days. When measuring the time taken to return to pretreatment levels we assessed data from the washout periods only. PEF records were available only following withdrawal of treatment after the first or second treatment periods. PEF response was calculated as a percentage change in mean PEF during the final seven days of treatment from mean pretreatment PEF.

\section{Results}

\section{PREDNISOLONE}

Fifty of the 121 patients showed a full or partial response to oral prednisolone. Of these, $32 \mathrm{had}$ a detectable increase in PEF as defined and their data were analysed further. In only 18 instances was a record of PEF available for analysis after withdrawal of treatment. The
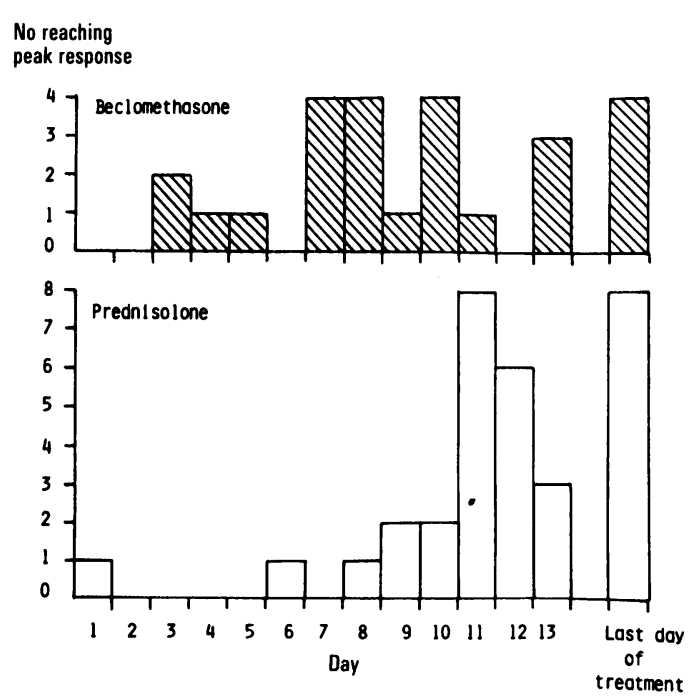

Figure 2 Histogram showing the day on which the peak response in mean daily peak expiratory flow (PEF) was reached in all 57 responses analysed for beclomethasone (hatched columns) and prednisolone (plain columns). 
Baseline characteristics of the responses with and without records available for the determination of duration of effect of treatment

\begin{tabular}{lll}
\hline & $\begin{array}{l}\text { Data } \\
\text { available } \\
(n=36)\end{array}$ & $\begin{array}{l}\text { No } \\
\text { data } \\
(n=21)\end{array}$ \\
\hline $\begin{array}{l}\text { Day of peak effect for response } \\
\text { (median[range]) }\end{array}$ & 11 & 11 \\
$\begin{array}{l}\text { Pretreatment peak flow } \\
(1 / \text { min, mean (SD)) }\end{array}$ & $223(82)$ & $204(91)$ \\
$\begin{array}{l}\text { Percentage increase in peak flow } \\
\text { with response } \\
\text { (mean (SD)) }\end{array}$ & $16 \cdot 2(11 \cdot 1)$ & $14 \cdot 6(10 \cdot 0)$ \\
\hline
\end{tabular}

time to peak effect with oral prednisolone varied from one to more than 14 (median 12) days (fig 2). In eight of the responses the daily mean PEF was still rising on the final day of the treatment period.

\section{BECLOMETHASONE}

Forty two patients had full or partial responses to inhaled beclomethasone, of whom 25 had a detectable increase in PEF over the treatment period; 18 of these had a record for duration of action analysis. Response to inhaled beclomethasone reached its peak effect at times ranging from three to more than 14 (median 9.5) days, four responses showing the peak daily mean PEF on the final treatment day.

In total, 44 patients showed a response to active treatment on 57 occasions. The time to peak response with inhaled treatment was shorter than with oral prednisolone (Wilcoxon rank sum test: $\mathrm{n}_{1}=32, \mathrm{n}_{2}=25, \mathrm{Z}=2.51$; $\mathrm{p}<0.01$ ). Thirteen patients had a response to both treatments; the analysis produced the same conclusions if these patients were removed (median (range) days: beclomethasone 8(3-14); prednisolone 11(1-14); Wilcoxon rank sum test: $n_{1}=19, n_{2}=12 ; Z=2 \cdot 43 ; p<$ $0.01)$.

The effect of treatment was still apparent in half $(18 / 36)$ of the responses 14 days after treatment had been withdrawn, in that the daily mean PEF had not returned to pretreatment levels. The proportion of responses in which daily mean PEF was still above pretreatment levels at 14 days was similar for beclomethasone and prednisolone (beclomethasone 7/18; prednisolone $11 / 18 ; \chi_{1}^{2}=1 \cdot 78 ; \mathrm{NS}$ ).

\section{PLACEBO}

A full or partial response to placebo treatment occurred in 24 of the 107 patients, though only five showed a significant increase in daily mean PEF. The PEF response to placebo was less than the PEF response to the two active treatments $\left(\chi_{2}^{2}=12 \cdot 1, \mathrm{p}<0.01\right)$. Peak effect occurred on days $5,7,10$, and 11 (two subjects), and the time taken to return to pretreatment levels (available for three responses) was one day (two cases) and five days.

The two observers agreed in their assessments in all but two cases, differing in these by one day only (in the analysis the lower value has been used).

The characteristics of responses with and without records available after withdrawal of treatment are shown in the table. There are no significant differences.

\section{Discussion}

Our results show that the time course of response to oral and inhaled corticosteroids varies considerably and that two weeks' treatment is not long enough to ensure that maximum improvement is attained in all patients. In addition, our analysis indicates that the effect of such treatment may persist for longer than two weeks in many patients.

We have not been able to study the time course of response in all patients we classed as responders to treatment. In patients responding in terms of their $\mathrm{FEV}_{1}$ or FVC or both we were able to analyse the time course of response only if a significant improvement in PEF occurred over the treatment period. We have tried to eliminate chance variation in PEF by using a statistical assessment of PEF changes over the treatment period to select responses for further graphical analysis. As definition of response state is related to baseline measurements and not to pretreatment measurements, some responders may show no significant improvement in PEF when their data are analysed in this way. Responders to placebo produced statistically fewer responses for graphical analysis, however, suggesting that our analysis is looking at the true time course for active treatment.

Previous studies on the effect of oral corticosteroids on non-asthmatic chronic airflow obstruction have generally used two week treatment periods, in accordance with the work of Webb et al. ${ }^{4-12}$ With such a design a few responders may be missed, and the peak response attainable in an individual may not be reached in up to $20 \%$ of patients. The chosen length of treatment in such trials must balance this aspect with the suppression of the hypothalamic-pituitary-adrenal axis, which is detectable biochemically at three weeks. ${ }^{13}$ In many patients with asthma inhaled corticosteroids do not produce maximum benefit in many patients until four to six weeks after treatment is begun. ${ }^{14}{ }^{15}$ Our results are compatible with a similar time course in patients with airflow obstruction not due to asthma, and too short a period of treatment may explain the disappointing results obtained by Harding and Freedman in a previous study. ${ }^{6}$ Surprisingly, inhaled beclomethasone produced a peak response more quickly than oral prednisolone in our patients. One reason for this may be a difference in the degree of response achieved. A drug producing a smaller improvement would produce the peak response earlier if the rate of improvement were similar. The increase in PEF with inhaled beclomethasone (13\%) was less than that seen with oral prednisolone $(17 \cdot 6 \%)$.

The prolonged beneficial effect of treatment explains the placebo order effect noted in our study. ${ }^{5}$ Benefit from treatment may last for over two weeks after it is withdrawn, but because 21 of 57 responses $(37 \%$ ) did not have 
records available for duration of effect analysis our conclusions must be cautious. The responses where records were available for analysis did not differ significantly in any way from those without records after the end of treatment (table), suggesting that our results are valid. Our analysis of the duration of effect of treatment may give an underestimate. In five responses in which a placebo treatment period followed the 14 day washout period the mean daily PEF remained above the baseline period for $22,23,28,41$, and 42 days. A similar prolonged improvement in pulmonary function has recently been shown in patients with chronic asthma after withdrawal of oral prednisolone. ${ }^{16}$ In this study nearly half of the group taking placebo after a short course of oral prednisolone had no major deterioration in symptoms and pulmonary function 28 days after prednisolone was withdrawn.

A treatment order effect has not been seen in previous trials of oral corticosteroids in similar patients, though only two reports have specifically commented on this. With their smaller number of patients these trials may have lacked the statistical power to detect this effect. Our results suggest, however, that to avoid this trials should incorporate a washout period of well over two weeks. If this is impracticable in the clinic setting a single blind or observer blinded sequential assessment may be more appropriate.
1 Anonymous. Steroids for bronchitic exacerbations. Lancet $1981 ; \mathrm{i}: 84$.

2 Anonymous. Steroids in bronchitis. $\mathrm{Br}$ Med J 1980;281: 1088-9.

3 Sahn SA. Corticosteroids in chronic bronchitis and emphysema. Chest 1978;73:389-96.

4 Webb J, Clark TJH, Chilvers C. Time course of response to prednisolone in chronic airflow obstruction. Thorax 1981; 36:18-21.

5 Weir DC,Gove RI, Robertson AS, Burge PS. Corticosteroid trials in chronic airflow obstruction. A comparison of oral prednisolone and inhaled beclomethasone dipropionate. Thorax 1990;45:111-6.

6 Harding SM, Freedman S. A comparison of oral and inhaled steroids in patients with chronic airflow obstruction: features determining response. Thorax 1978;33:214-8.

7 Mitchell DM, Rehan R, Gildeh P, Dimond AH, Collins JV Effects of prednisolone in chronic airflow limitation. Lancet 1984;ii:193-6.

8 Mitchell DM, Gildeh P, Dimond AH, Collins JV. Value of serial peak expiratory flow measurements in assessing treatment response in chronic airflow limitation. Thorax treatment response

9 Mendella LA, Manfreda J, Warren CPW, Anthonisen NR. Steroid response in stable chronic obstructive pulmonary disease. Ann Intern Med 1982;96:17-21.

10 Lam WK, So SY, Yu DYC. Response to oral corticosteroids in chronic airflow obstruction. Br J Dis Chest 1983, 77:189-98.

11 Stokes TC, Shaylor JM, O'Reilly JF, Harrison BDW. Assessment of steroid responsiveness in patients with chronic airflow obstruction. Lancet 1982;ii:345-8.

12 Eliasson O, Hoffman J, Trueb D, Frederick D, McCormick JR. Corticosteroids in COPD. Chest 1986;89:484-90.

13 Webb J, Clark TJH. Recovery of plasma corticotrophin and cortisol levels after a three week course of prednisolone. Thorax 1981;35:22-4.

14 Clark TJH. Effect of beclomethasone dipropionate delivered by aerosol in patients with asthma. Lancet 1972;ii:1361-4. 15 Mintz S, Broder I, Davies G, Leznoff A, Rostum H, Silverman F. A double blind crossover study of beclomethasone dipropionate in asthmatic patients with and without chronic bronchitis. Postgrad Med J 1975;51 (suppl 4):76-9.

16 Salemeron S, Guerin J-C, Godard P, et al. High doses of inhaled corticosteroids in unstable chronic asthma: a multicentre, double-blind, placebo controlled study. $A m$ Rev Respir Dis 1989;140:167-71. 